\title{
What proportion of patients admitted with stroke or TIA may be suitable for newer cholesterol-lowering treatment? (ESOC8-0628)
}

\section{St George's University Hospitals W/HS NHS Foundation Trust}

Vafa Alakbarzade \& Anthony C. Pereira.

St George's University Hospitals NHS FT, London, UK

\section{Background}

Raised serum low-density lipoprotein cholesterol (LDL-C) and non-high-density lipoprotein cholesterol (non-HDL-C) are associated with increased risk of cerebrovascular disease ${ }^{1,2}$. Cerebrovascular benefit from LDL-C and non-HDL-C lowering has been demonstrated with statins ${ }^{1}$. However, a proportion of patients with ischaemic stroke or TIA are either high-dose statin resistant or intolerant. The FOURIER trial showed the anti-PCSK9 agent, Evolocumab when added to statin therapy, further lowered LDL-C and significantly reduced the risk of cardiovascular events ${ }^{3}$.

NICE guidelines recommend the use of Evolocumab for primary, non-familial hypercholesterolaemia in ischaemic stroke patients if LDL-C is persistently $>4.0 \mathrm{mmol} / \mathrm{l}$ or $3.5-3.9 \mathrm{mmol} / \mathrm{l}$ with poly-vascular disease. For patients with familial hypercholesterolaemia $(\mathrm{FH})$ and ischaemic stroke, Evolocumab is recommended if the LDL-C concentration is persistently $>3.5 \mathrm{mmol} / \mathrm{l}$.

In our current study, we evaluated stroke admissions potentially eligible for more intensive cholesterol treatment.

\section{Methods}

$>$ We performed a retrospective evaluation of consecutive cases within a single, regional, hyperacute stroke unit at St George's Hospital, the main referral centre for south-west London.

- We reviewed consecutive medical and laboratory records for all acute ischaemic stroke (AIS) and TIA patients presenting to the service from January 1st 2016- June 1st 2016.

$>$ Records were hand-searched by and reviewed by the authors.

$>$ Data were collected on diagnosis, risk factors and stroke work-up.

$>$ The Dutch Lipid Clinic Network Algorithm for Familial Hypercholesterolemia (FH) screening and TOAST classification were used.

\section{Results}

We achieved $100 \%$ case record ascertainment of 650 patients admitted.

$351(54 \%)$ had acute ischaemic stroke (AIS) or TIA, 80(12\%) haemorrhage and 219(34\%) mimic syndromes. Patients with haemorrhage, mimic syndromes and absent LDL-C or non-HDL-C testing were subsequently excluded.

Of 324 AIS/TIA patients: 6 had possible FH (LDL-C >5.0 mmol/l); 23 had LDL-C $>4.0 \mathrm{mmol} / \mathrm{l}$ or $3.5-3.9 \mathrm{mmol} / \mathrm{l}$ and concomitant very high vascular risk ( $>1$ vascular bed involved), and 21 had non-HDL-C $>3.4 \mathrm{mmol} / \mathrm{l}$ and recurrent cardiovascular events.

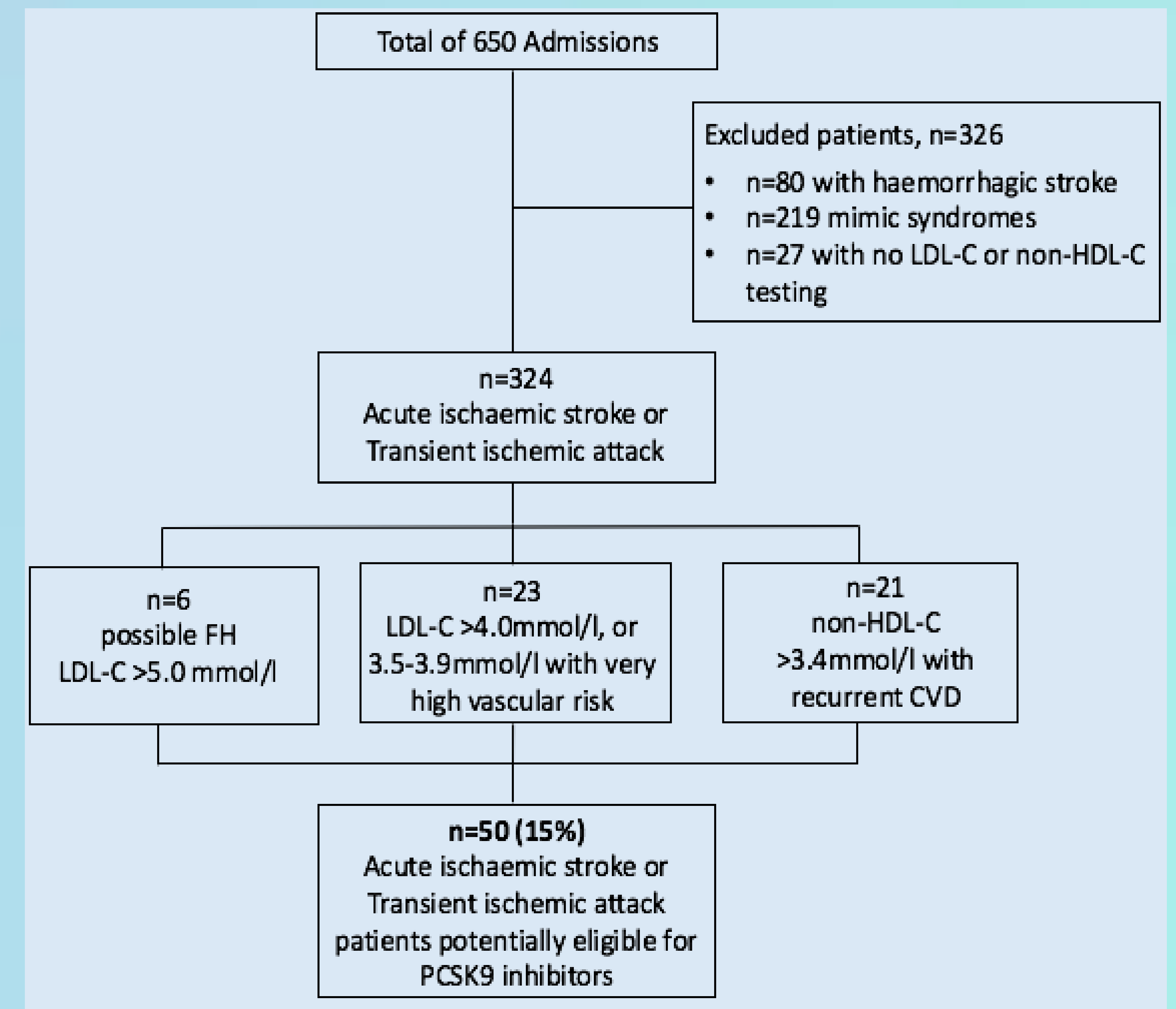

Median vascular risk factors per AIS or TIA patient potentially eligible for PCSK9 inhibitors were 2 (0-5 IQR), similar to the non-eligible AIS or TIA cohort. $50 \%$ had a history of recurrent cerebrovascular events versus $31 \%$ in treatment non-eligible AIS/TIA cohort, $p=0.007$.

\begin{tabular}{l|cc}
\hline TOAST classification & $\begin{array}{c}\text { Treatment } \\
\text { eligible } \\
\text { AIS/TIA } \\
(\mathbf{n = 5 0 )}\end{array}$ & $\begin{array}{c}\text { Treatment } \\
\text { non-eligible } \\
\text { AIS/TIA } \\
(\mathbf{n = 2 7 4 )}\end{array}$ \\
\hline \hline Cardioembolic, No. (\%)* & $74.3(12)$ & $72.5(15)$ \\
Lacunar syndrome, No. (\%)** & $22(44)$ & $136(50)$ \\
\hline Carotid artery stenosis $>70 \%$, No. (\%)*** & $20(40)$ & $150(55)$ \\
Other determined aetiology, No. (\%) & $8(16)$ & $81(30)$ \\
\hline * Based on comorbid atrial fibrillation or new atrial fibrillation detected on 12 lead ECG and MRI findings
\end{tabular}

Bed on comorbid atrial fibrillation or new atrial fibrillation detected on 12 lead ECG and MRI findings

**Based on MRI findings after exclusion of other causes

****Based on Doppler carotid scan or CT angiography

Characteristics

\begin{tabular}{cc}
$\begin{array}{c}\text { Treatment } \\
\text { eligible } \\
\text { AIS/TIA } \\
(n=50)\end{array}$ & $\begin{array}{c}\text { Treatment } \\
\text { non-eligible } \\
\text { AIS/TIA } \\
(n=274)\end{array}$ \\
\hline $74.3(12)$ & $72.5(15)$ \\
$22(44)$ & $136(50)$ \\
$20(40)$ & $150(55)$ \\
$8(16)$ & $81(30)$ \\
$14(28)$ & $82(30)$ \\
$17(34)$ & $56(20)$ \\
$4(8)$ & $16(6)$ \\
$8(16)$ & $36(13)$ \\
\hline
\end{tabular}

Age, mean (SD), y

Male sex, No. (\%)

Hypertension, No. (\%)

Diabetes mellitus, No. (\%)

Atrial fibrillation*, No. (\%)

Ischaemic heart disease, No. (\%)

Peripheral vascular disease, No. (\%)

Smoking, No. (\%)

*Atrial fibrillation detected on 12 lead ECG

\section{Discussion}

We aimed to determine the proportion of patients in an in-patient cerebrovascular disease service who may be eligible for more intensive cholesterol treatment. About half of the treatment eligible patients included acute ischaemic stroke or TIA patients with persistently high non-HDL-C levels with recurrent cardio-vascular disease. NICE recommendations only include LDL-C for Evolocumab eligibility. However, serum non-HDL-C levels are an independent risk factor of ischemic stroke and Evolocumab is also associated with significant reduction of other atherogenic lipids including non-HDL-C $\mathrm{C}^{2,4}$.

\section{Conclusion}

Up to $15 \%$ of unselected acute ischaemic stroke or TIA patients were potentially suitable for anti-PCSK9 agents. Our data may guide sample size selection for stroke trials testing these new drugs.

\section{Acknowledgements/Funding}

AMGEN group for funding of ESOC travel (VA)

\section{References}

1. The Stroke Prevention by Aggressive Reduction in Cholesterol Levels (SPARCL) Investigators High-dose atorvastatin after stroke or transient ischemic attack. N Engl J Med. 2006;355:549-559

2. Wu J et al. Non-high-density lipoprotein cholesterol vs low-density lipoprotein cholesterol as a risk factor for ischemic stroke: a result from the Kailuan study. Neurol Res. 2013 Jun;35(5):505-11. doi: 10.1179/1743132813Y.0000000206. Epub 2013 Apr 16.

3. Sabatine MS et al; FOURIER Steering Committee and Investigators. Evolocumab and clinical outcomes in patients with cardiovascular disease. N Engl J Med. 2017;376:1713-1722. doi:

10.1056/NEJMoa1615664

4. Rosenson RS et al., Efficacy and Safety of the PCSK9 Inhibitor Evolocumab in Patients with Mixed Hyperlipidemia. Cardiovasc Drugs Ther. 2016 Jun;30(3):305-13. doi: 10.1007/s10557-016-6666-1. 